\title{
Overcoming methodological challenges in prevalence studies in developing contexts with
} vulnerable children

\begin{abstract}
Social work research concerns itself with vulnerable populations. Methodological challenges including accessing vulnerable populations are especially acute in developing countries where systematized data is often lacking. This article presents a pilot study using Respondent Driven Sampling (RDS) to estimate the prevalence of children engaged in commercial sexual exploitation. The results are used to illustrate the feasibility of developing multi-sectoral collaborations to address issues among vulnerable populations. This study demonstrates that RDS is a strong design capable of producing prevalence estimates. Implications for international social workers in facilitating professional capacity-building for effective planning, monitoring, and reporting of social development projects are discussed.
\end{abstract}

Keywords: Methodology; Respondent Driven Sampling; Child Commercial Sexual Exploitation; International Social Work 


\section{Overcoming methodological challenges in prevalence studies in developing contexts with}

vulnerable children

Introduction

True to its values base and purpose, social work research focuses its attention on extremely vulnerable populations such as sexually exploited children and youth. However, this is not without considerable challenges including, notably, gaining access to these young people. Mainstream survey methods for estimating prevalence may fail to deliver an accurate picture of the actual population of interest. These challenges are especially acute in developing countries and when dealing with sensitive and 'hidden' problems such as commercial sexual exploitation of children (CSEC). Gaining insight into the prevalence and context of such problems is critical to social workers in order to provide evidence-based advocacy, policy and intervention. The main purpose of this paper is to examine the application of a specific research method, respondent driven sampling (RDS), through the critical examination of the protocol, implementation and analysis of data from a pilot study conducted on CSEC in the Kathmandu Valley, Nepal.

Estimating prevalence of hidden populations is a global challenge requiring the utilization of sound methodology to determine the size of the affected population. In developing contexts, these methodological issues may be particularly complex as statistical systems and methods often are comparatively less sophisticated and systematized (Belkindas and Swanson, 2014). Where local governments are weak and/or corrupt, international and local nongovernmental organizations (NGOs) often become alternative sources of social service and welfare provision (Ulvila and Hossain, 2002). NGOs can play an important role in providing and shaping service development for vulnerable population groups, as well as assisting in building 
capacity in rigorous research and evaluation methods within global international development contexts (Suárez and Marshall, 2014; Bhatta, 2013; Harwin and Barron, 2007). Within such contexts, international social workers can play a critical role in supporting capacity building in methodology and the use of evidence-based research for advocacy practice.

This study examined the feasibility of using Respondent-Driven Sampling (RDS) as a method to estimate the prevalence of commercial sexual exploitation of children in Nepal. The structure of the paper is as follows. First, we briefly describe the research context and partnership approach. Second, we describe the methodological approach including discussion of research ethics. We present illustrative findings to demonstrate the implementation potential of the methodology. In closing, we evaluate how adopting the RDS method can build research-based evidence in developing contexts such as Nepal and inform civil society and state responses. Finally, we draw out implications for international social work professional practice more broadly.

\section{Background and Research Context}

\section{Academic-Field Partnership}

This study was conducted in partnership by a team of international social work academics and one International Non-Governmental Organization (INGO) Nepal country office, supported by funding from the international parent organization of the INGO. The partnership was crucial for linking together the research consultants and grassroots local NGOs and ensuring culturally aware implementation. Field-academic partnerships present unique opportunities as well as challenges (Regehr, Stern, and Shlonsky, 2007), as some of the ethical stances and practices inherent in the scientific research process (neutrality and objectivity) may be in conflict with 
professional practice codes of ethics (intervention). Furthermore, the timelines and goals of academic research may conflict with the reality of field practices (Bartunek and Rynes, 2014). Indeed, and more specifically, this challenge is concerned with the privileging of certain knowledge epistemologies over others, where the scientific method and process of research is held up as the gold standard (MacIntyre and Paul, 2013). Social work research practitioners are particularly well-suited to address these types of ethical dilemmas given our professional training and codes of ethics.

We will discuss some of the specific ethical issues we confronted in the study implementation, especially in relation to conducting research with vulnerable children after an initial introduction to the research context.

\section{Nepalese Context of CSEC}

Many countries including Nepal do not have adequate administrative data systems that can provide reliable standards for measuring the magnitude of the problem of CSEC (WHO, 2016; ILO, 2007). The exact number of male and female children involved in CSEC in Nepal is unknown. The National Centre for AIDS and STD Control estimates there are 30,815 commercial sex workers in Nepal and comparative research suggests between $33 \%$ and $50 \%$ of these workers are children (TdH, n.d.). The presence of boys and men in CSEC and the entertainment sector is visible and well known, but largely under-recognised and researched.

We defined commercial sexual exploitation of children (CSEC) following the Stockholm Declaration from the World Congress against Commercial Sexual Exploitation of Children (1996) as a fundamental violation of children's rights comprising sexual abuse by the adult and remuneration in cash or kind to the child or a third person or persons. In industrialized and 
developing countries alike, worst forms of child labour including CSEC are recognized as acts of violence against children, and Nepal is no exception to this. Indeed, the World Health Organization (2016) recognized commercial sexual exploitation of children as one of the most urgent areas that need to be addressed.

Prostitution is illegal in Nepal. However, even with legal restrictions and regulation protocols, the adult entertainment industry in Nepal is facing rapid growth and so is the number of children who are involved in it. In the Kathmandu Valley, an estimated 11,000 to 13,000 adult women and girls are working in the night entertainment industry (Frederick et al., 2010). Nearly one half of all entertainment workers entered the industry before the age of 18 (Ibid). Not all children involved in the Nepal entertainment sector are involved in CSEC, however, undeniably, the risks and general environment provide compelling factors towards a gradual or forced immersion into the sex trade for some (ECPAT, 2015).

The Trafficking in Persons Report 2013-15 (2016) states that 8-8,500 people in the full year of 2013/14 were trafficked to India from Nepal. Note this is not restricted only to girls and includes all people. Studies in Nepal have reported that Nepalese children are often trafficked domestically for various purposes including commercial sex as well as child soldiering, circus entertainment, factory work, and domestic servitude (Deane, 2010; Sacouman, 2012). Internationally, Nepalese children are trafficked predominately to India and the Middle East for commercial sex and/or forced marriage (Ibid.). Within the South Asian region, Nepal is the country with the highest prevalence of child and women trafficking, followed by India (Deane, 2010). The volume of child sex trafficking between Nepal and India has also been increasing in recent years and reflects the growing sophistication of organized crime in the region. Although dated, an older study describes two types of sex trafficking in Nepal: the first is "soft trafficking" 
where girls are sold off to brothels by family members in desperation, or under the pretense of marriage and employment opportunities, while the second is "hard trafficking", where children are abducted or coerced into engaging in commercial sexual activities (Holmstrom, 1999).

These various sources offer some insight into the scope of child trafficking and a crude indication of populations at risk of CSEC through trafficking and involvement in the adult entertainment services in Nepal. There remains, however, a notable dearth of empirical studies and exploration of appropriate methodologies to estimate the actual prevalence rates as well as a general lack of systemized, accurate, or reliable information. A lack of accurate data inhibits advocacy capacity to local authorities and government and hampers the efforts of NGOs to formulate strategies, intervention, and appropriate social services for these children (Sacouman, 2012). This paucity of information is one of the primary motivations for the current pilot study.

\section{Methodology}

The primary research method implemented for the study was Respondent Driven Sampling (RDS). RDS technique is a chain-referral method, considered superior to standard snowball convenience sampling methods (McCreesh et al., 2012). RDS is used to obtain statistically representative samples of hard-to-reach groups which take into account the social connections (social network) among the target group members. Obtaining statistically representative estimates allows for reduced bias known to snowball techniques. The method has been successfully implemented with street children (Johnston et al., 2010) and research findings indicate the relative value of using the RDS method over other methods commonly implemented with similarly 'hidden' populations (McCreesh et al., 2012). The method has potential broad relevance for domestic as well as international social work professionals and researchers as many social work populations can be described as 'hidden'. 
RDS involves strict adherence to specific field implementation and post-hoc statistical analytic procedures which account for the non-random recruitment patterns of the sample. The protocol adherence and subsequent analytical techniques allow for adjustment of the prevalence estimates to approximate a random sampling approach. Without some type of adjustment, the likelihood of a similarity bias based on common characteristics to a social network inherent in the snowball sample approach could lead to a higher estimate of prevalence than is actually present in the wider target population. We discuss in turn the key elements of the method.

\section{Seed Respondents}

The field procedure starts with the identification of "seed" respondents who are given coupons (typically three) to recruit others from the target population. These initial participants are crucial as they are relied upon to lead the recruitment among their social networks. An ideal seed is an individual who is well connected within the target networks (among their peers), well regarded by peers, sympathetic to the survey's goals and diverse with regards to key target characteristics (Johnston and Sabin, 2010; McCreesh et al., 2012). In our study the seeds were chosen to reflect the diversity of social networks incorporating information about the target population from prior research and practice knowledge consultation with local experts. The consulting team held discussions with the key partner INGO and local implementing NGOs to introduce the study and decide on the characteristics for selecting the seeds. Seeds were selected from each of the major subpopulations based on consultation and the proposed eligibility criteria.

The eligibility criteria for the study differed for boys and girls based on practice knowledge about the divergent patterns of CSEC among boys and girls in Kathmandu (Frederick et al., 2010). Generally, girls work in venues which are formally or informally a part of the adult entertainment services, such as entertainment clubs, bars, restaurants or massage parlours, 
although some are working in street-based prostitution. Boys' involvement in CSEC is distinct. Exploitation tends to take place through networks of men and youth, and often occurs through initial contact in public places or through social and electronic media and takes place in private abodes and guest houses. These locations are further known as areas where men who have sex with men (MSM) associate. Males involved in CSEC were often non-heterosexual in orientation and this is an added vulnerability factor for boys entering the CSEC sector. The recruitment of the male seeds and subsequent participants focused on locations known to be sites frequented by boys involved in CSEC and gave special attention to monitoring potential issues of coercion and manipulation considered to be prevalent within male CSEC in Kathmandu.

A brief set of screening questions was used to identify respondent's eligibility for enrolment. The eligibility criteria for boys included: between the ages of 12 to 18 years inclusive, and having had at least one sexual partner. The eligibility criteria for girls included: between the ages of 12 to 18 years inclusive, and currently working in any one of a list of entertainment venue types. While not all of these venues are part of the adult entertainment services exclusively, they are known to cross-over (ECPAT, 2015). In addition to the eligibility criteria, the seeds were selected to reflect diversity of age within the 12-18 age band (younger and older), gender/sexual orientation (boys) and type of working venue (girls). There are no precise guidelines about the number of initial seeds, except that the decision should be guided by the targeted study sample size for a study (Salganik, 2006) taking into account diversity of eligibility criteria. As this was a pilot study to test the feasibility of the method, rather than to generate reliable prevalence estimates per se, a small number of seeds, four female and six male was selected.

\section{Coupons and Respondent Tracking}


The seed respondents were each given three coupons, which are tracked using a unique sequence of numbers to facilitate subsequent data analysis. The seed received instructions to give the coupons to others in the social networks identified in the brief survey. The information about social networks from the survey in combination with the tracking (through the coupon numbers and seed-initiated recruitment chains) is critical to the estimation process in RDS. The social network questions mirror the eligibility criteria, and the respondent must have had contact with the network members in the past 30 days. The coupons are then used for the respondent to give to potential recruits to enroll in the study. The participant recruiter becomes eligible to obtain secondary incentives for successfully recruiting new eligible participants.

The new recruits are invited in turn to become recruiters building the linked sample. Recruits are given a site specific and appropriate (i.e. non-coercive, small gift or refreshment) incentive for taking part in the study, and further incentives for successfully recruiting other eligible participants. This process continues in recruitment "waves" until a predetermined sample size is reached, or until the distribution of participant characteristics becomes similar between the recruitment waves (McCreesh et al, 2012; Salganik, 2006). As this is only a pilot study, the lessons learned from implementation of the field method and applicability for social work contexts broadly is the primary focus. The prevalence estimates are considered illustrative because the study was stopped after a predetermined -and brief - period of time, rather than relying on sample saturation which is necessary to produce reliable estimates of a population using RDS.

\section{Incentives}

All participants were eligible for cash incentives for their participation. The use of incentives, although common in much global research, remains controversial. One of the key 
tenets to ethical research practice and voluntary consent is to ensure freedom of choice to participate (Grant and Sugarman, 2004). The use of an incentive could be considered coercive if, for example, the monetary value of the incentive is disproportionate to the material status of the potential research participant (Belfrage, 2016). Additionally, research with children highlights the potential risk of power differentials between researcher and child which could result in a type of social desirability bias: in effect a type of coercion, if the child perceives the need to fulfill social obligations of respect for adults (Alderson and Morrow, 2011; Baker and Charvat, 2008).

The use of incentives is a core component of the RDS method since not only is the research seeking individual participation, but further asking the participant to recruit and bring in additional respondents (Johnston and Sabin, 2010). The research interviewers were trained by qualified social work research consultants and INGO child rights professional staff. Training included material on the responsibilities of ethical research practice with children. The interviews were conducted in private spaces located within local NGO partner agencies and the children were offered access to services and provided with a referral card for their follow-up. The team of two research interviewers adhered to the INGO child protection ethical guidelines for practice at all times.

In the study implementation, a child was eligible to receive a primary incentive if $\mathrm{s} / \mathrm{he}$ met the screening criteria and completed three tasks: voluntary ethical consent; face-to-face interview; and receiving explanation of the coupon recruitment process. The primary incentive was set at 500 rupees (approximately US\$5) with an additional payment up to 300 rupees (approximately US\$3) for travel to the study site. Participants also received three coupons for future participant recruitment. The children were eligible for a secondary incentive if they met two conditions: (1) they returned to meet with the study team bringing their section of the 
coupon stub; and (2) if one or more of their recruited participants had screened as eligible for study participation. A secondary incentive of 500 rupees (approximately US\$5) per successful recruit could be claimed by all participants, with up to a total of 1500 rupees (approximately US\$15) additional incentives for each participant.

Selecting a level of incentive payment aimed to strike a balance whereby target population members deemed the research activity worthy of their time and effort, while remaining low enough that it did not encourage involuntary participation because of a high temptation for monetary gain. The incentive level was decided in consultation with local experts and deemed acceptable in order to increase the chances of successfully recruiting enough participants within the target population, which would allow for an overall assessment about the feasibility of the RDS methodology in the local context.

\section{Questionnaires}

The questionnaires were developed in partnership with the INGO whose representatives have had extensive experience dealing with CSEC in a culturally-, and gender-sensitive manner in the region. The screening criteria differed for the male and female populations taking into account diversity in work locations (e.g., venue (girls) versus street-based (boys)) as well as recognized differences between males and females in openness about discussion of sexual behaviours and activities. Basic information about age, employment status and living arrangements was collected from the boys. The girls were asked about general work activities before being asked about different types of sexual/sexualized activities. Questions about social networks were included to elicit estimates of other individuals known to the respondents who met the eligibility criteria and had been seen in the past month. 
An indirect method of asking girls about sexual practices in the workplace was used specifically to avoid having them talk directly about the sexual work activities which are considered culturally taboo (Marthur, Malhotra, and Mehta, 2001). The girls were asked an initial question about work activities broadly and asked to respond by providing the numbers corresponding to images of different common work activities. If the girl provided the number indicating any sexual activities, a second card with more specific details was provided, and the girl was asked to look at the card and give corresponding numbers repeating a similar method of identification. Whether the girl has experienced CSEC is determined by the responses to the second activity card. It was considered more culturally acceptable to directly ask about sexual engagement with boys. However, indirect methods of information gathering were also used with the boys to determine whether their experiences of CSEC were with male partners. This approach was underpinned by a similar rationale as that for the girls as a result of social taboos associated with such forms of activity. The boys were first asked about whether they know of the activity of boys/men having sex with boys/men, then if they know anyone who participates in this, and finally, if they have ever engaged in this themselves. Both of these indirect methods have been previously implemented by the team with similar populations in prior research (Frederick et al., 2010) and reflect more broadly methods of communication about sensitive topics and especially with children and young people who may be less likely to discuss personally intimate topics directly (Kolucki and Lemish, 2011; Malhotra and Mehta, 2001). All of the participants were provided with a small card with referral details at the end of the interview, and as the interviews were conducted in local NGO offices, access was immediate if necessary. Full ethical approval for the study was granted by the Griffith University Ethics Committee. 


\section{Results}

In the following section, we provide an overview of the sample and present the prevalence estimates based on sampling adjustment. We present further details about analytical procedure and the limitations of the current estimates given the small sample size of the pilot study.

\section{Sample profile}

Using the RDS method, a total of 94 male children respondents were recruited, 6 of whom were 'seeds' while 88 were additional recruits received through the standard procedures of referral. All 94 male children respondents had some prior experience of sexual activity with another person. Basic information about age, employment status and living arrangements was collected from the boys. One half of the male sample reported their age as $17(50 \%)$, with an additional $30.9 \%$ aged 18 . Two respondents were recorded within the age range of 12 to 18 , but withheld their exact age. Other key characteristics included information about employment status, living arrangements as well as sexual risk information including number of sex partners, gender and gender exclusivity of sex partners, and knowledge about MSM (See Tables 1 and 2).

\section{[Insert Tables $1 \& 2$ here]}

As for female children, a total of 133 girls between the ages of 12 to 18 participated in the survey. In the data cleaning process, eight cases were dropped because of inaccurate recording of respondent recruitment numbers, while three cases were dropped because they were linked by recruitment chain to one of the other dropped cases. Our analysis for the girls was conducted with a final sample size of $\mathrm{N}=120$ having eliminated the seed respondents. As illustrated in Table 1, the majority of the girls (87\%) were aged 16 to 18 . A little over $40 \%$ were 
working in dance bars, with an additional $24 \%$ working in 'Dohori' restaurants where food, drink and folk music services are offered, and the remaining $40 \%$ working across the spectrum of entertainment venues. The girls reported general work activities and different types of sexual/sexualized activities if they had experience of these in the workplace (See Table 3). The key outcome variable 'explicit CSEC' for the girls was based on a series of questions about any intimate touching, masturbation, oral sex or vaginal intercourse as a part of a girl's work place or work contacts. This is a conservative definition of CSEC compared to the definition adopted by $\mathrm{I} /(\mathrm{NGOS})$ including those collaborating in this study to reflect what is considered severe CSEC. The unadjusted response rate (count of actual sample responses) for 'explicit CSEC' was 94.4\% among the full sample of girls.

[Insert Table 3 here]

\section{Network and Prevalence Estimates}

The RDS method relies on the use of social network data to adjust the resulting prevalence estimates. The strength of RDS is that it can reduce some of the biases inherent in chain referral (snowballing) sampling methods. The use of a probability sampling method, produces estimates to measure the precision of the results, which can have important implications for subsequent service delivery and/or advocacy. A relatively small sample size influences the precision of the prevalence estimates, as some researchers indicate that the RDS method should rely on larger sample sizes than traditional probability methods (Johnston and Sabin, 2010). The prevalence estimates for CSEC by gender for the current study were generated using RDSTAT 7.1 software. Within the male and female subsamples two prevalence estimates by age band were generated, taking into account the age distribution of the samples. As the boys were on average older than the girls, the age brackets included boys (1) age 17 and under; and 
(2) age 18, whereas for the girls the age brackets were age (1) 16 and under; and (2) 17 and 18. The estimates and related measures are discussed separately by child gender.

\section{Boys}

The outcome variable measuring prevalence for the boys is based on the question "Do you trade or have traded sexual activities with other boys (or men?) for gain or benefits?" After accounting for the design features of the RDS sample, the proportions of boys estimated within each age group were 21.9 (younger) and 78.1 (older), and the average social network size for two groups of boys varied 3.35 (younger) compared to 4.32 (older). This information is used to generate the prevalence estimate of CSEC among less than age 17 at $20 \%$ with the confidence interval of $8.5 \%$ to $34.6 \%$. The prevalence estimate for the older boys aged 18 is almost four times as much at $77.1 \%$ with the confidence interval of $60.6 \%$ to $89.2 \%$. The confidence intervals are quite wide, likely reflecting the relatively small sample size and the smaller number of recruitment waves imposed by the pilot study design.

\section{Girls}

The outcome variable 'explicit CSEC' for prevalence of CSEC of the girls respondents was based on the recoding of a question where girl respondents reported any intimate touching, masturbation, oral sex or vaginal intercourse as a part of her work place or work contacts as described above.

Based on the eligibility criteria the social networks of the girls consisted of "[other] girls aged 12-18 who work in target areas and venues whom the respondent has seen in the past month". After accounting for the design features, the proportions of girls estimated within each age group were $19.7 \%$ (younger) and $80.3 \%$ (older), and the average social network size for the 
two groups of girls varied 2.69 (younger) compared to 4.37 (older). This information is used to generate the prevalence of CSEC among girls with resulting figures of $16 \%$ with confidence intervals of $8.2 \%$ to $25.2 \%$ (younger) and $73.8 \%$ with confidence intervals of $60.9 \%$ to $86.3 \%$ (older). Similar to the male sample, the confidence intervals are fairly wide.

In both instances, the pilot study highlights troubling trends in the CSEC of male and female children and young adults (age 18) in Kathmandu, Nepal. Even if the lower bound of the confidence interval is taken as indicative of the prevalence of CSEC, this translates into a sizeable number of children (under age 18) who are experiencing exploitative and harmful sexual contact as a part of their formal (females) and informal (males) employment/livelihood activities. Additionally, the estimates for the older boys and girls in the sample, ages 17 and 18 is particularly alarming, with the lower bound of both confidence intervals for the older age groups at over $60 \%$ prevalence of CSEC.

\section{Discussion and Implications for Practice}

The primary purpose of this study was to explore the feasibility of the RDS method for this sample group - female and male Nepalese children in CSEC and determine the suitability of this method for gathering data about a very vulnerable child population in a country with fragile economic, governmental and civil society structure. The method has broad applicability for social work practitioners globally who work with hidden populations in developing countries.

The indication of high prevalence rates of problems such as CSEC based on empirically supported studies ultimately highlights the need for governments to provide the necessary resources to intervene. Unfortunately, due to limited prevalence data in countries like Nepal, CSEC can remain a relatively low prioritized area owing to a lack of awareness of the breadth of 
the problem in concert with competing demands on scarce governmental resources. Such tensions are perhaps even more acute in some of the most controversial practice areas, and CSEC is one such area of practice. It is the very nature of these controversial areas with vulnerable populations that increases the importance of having reliable data to accurately target, and assess the impact of service interventions. As recommended by Spies et al (2015), a systematic framework for gathering and analyzing information pertinent to vulnerable children is imperative in developing any effective intervention. This pilot study highlights strong evidence of CSEC in the study areas amongst the target population. The prevalence estimates paint a picture of formal and informal employment/livelihood strategies that routinely expose children and young adults aged 18 to risky and exploitative sexual encounters. The successful implementation of the study, including the specific recruitment protocols, as well as the willingness of the respondents to share information about socially taboo practices suggests this method is promising for such endeavors. One of the incentives for conducting the pilot study was to investigate whether the young people would be willing to share the personal information required to researchers. Surprisingly, we found that the respondents were quite open, and at times it appeared that having the more neutral researcher to speak with (in contrast to a NGO worker) was beneficial for gaining trust. It may be that the relative neutrality of the researchers made the social taboo associated with the CSEC less restrictive.

There are a number of limitations in the implementation of the current study that must be recognized, and can be applied to future projects using a similar design. While the time-limited nature of the pilot study restricts the scope at which prevalence estimates can be generalized, the collected data highlights different levels of participation by age group of boys and girls. The data obtained from younger participants (especially boys) was more limited and estimates based on 
age groups need to be interpreted with caution. This has design implications for research instruments that record age especially when researching topics of sensitivity and illegality. Future studies should focus on recruiting initial seeds with stronger connections to the younger age brackets, and also consider having an option for recording age range (e.g., 12-15, 16-18) as a second-best option to precise age if the respondent is reluctant to disclose age.

While the small-scale study does not provide definitive population prevalence estimates, it does offer a call to action for further larger scale investigation of the prevalence of CSEC in the Kathmandu Valley and other locations where there is a lack of accurate information about the magnitude and scale of the problem. Failure to accurately assess vulnerable and at risk populations that are the target of international donor funding is a failure of the broader system to address critical issues of international child protection (Belkindas and Swanson, 2014; Suárez and Marshall, 2014). Indeed, appeals for funding both internally and externally for prevention and intervention has been justified with qualitative data and field experience. Whilst valuable, this data needs to be triangulated with statistical data that reveals the depth of the problem as well as providing baseline population estimates for future measurement of change (UNICEF, 2011).

Empirical evidence provides essential information to guide design and delivery of services, assess program outcomes and facilitate advocacy for greater systemic change including financial resources. This paper illustrates the relationship between research and service provision for an extremely vulnerable child population group, child commercial sex workers, in Nepal, and highlights the lack of available systematized information about the target population. To date, estimation of the prevalence of CSEC has often been speculative and relatively easy to contest or dismiss. The dearth of accurate data poses considerable hindrances. First, advocacy and 
representation to government, local authorities, and donors requires a strong empirical evidence base of the problem to justify action and allocation of resources. Second, accurate data is needed to strategize and plan the delivery of effective intervention strategies. In response to this knowledge gap, we employed the RDS method to test the feasibility of estimating the prevalence of male and female children engaged in CSEC. The study demonstrates that RDS is a promising method for gathering the necessary data needed for both understanding the size of the problem and systematic planning.

Field practitioners including social workers are increasingly recognized as playing a key role in supporting and working with these marginalized children and youth (O'Kane, 2002; Rigby and Whyte, 2015). Building capacity in service delivery and developing innovative approaches in terms of methodology and practice are important aspects for INGOs working in partnership with local NGOs as demonstrated by the current study. Targeting capacity building in service delivery is indeed one critical component of supporting developing countries to achieve milestones in addressing issues of children's rights and protection (UNICEF, 2011). Likewise, the continual knowledge exchange and dissemination of social work research and practice between the global North and developing countries must be strengthened (Pawar, 2015). However, the ability to comprehensively assess the impact of service delivery interventions is linked with the kind and amount of information that is available about the target service population. This requires not only a baseline such as rigorous prevalence estimation but also data that elucidates the scale of the problem.

In the short term, there are inherent limitations in purely assessing the impact of service delivery programs on individual children who are engaged in services using more in-depth case study methods. Such information is very useful for understanding the complexity of cases, 
monitoring service delivery and refining intervention models, and makes important contributions to policy advocacy. However, in the longer term, the absence of key information about the characteristics and size of the target population contributes to a lack of understanding about broader human rights and development agendas as little can be said about the impact of INGO interventions on the target sector without accurate prevalence data of the target population. Agenda setting which is frequently driven by well-meaning donors and funding agencies to provide ameliorative care and intervention that supports the rehabilitation and removal of children from exposure to conditions such as CSEC, often fails to consider the critical nature of more routine data collection that addresses the issue at a macro level. Within a child rights framework this is a necessity for understanding if objectives such as the Sustainable Development Goals are being progressed at a population level.

\section{Conclusion}

In conclusion, a number of key lessons to inform academic-field research collaboration emerged from the pilot study. First, the RDS method is a strong design capable of producing reliable prevalence estimates. A subsequent study which takes into account the key lessons learned is likely to contribute robust estimates of the prevalence of CSEC in Nepal. Second, this paper illustrates the relationship between research and service provision for one of the most vulnerable population groups in Nepal, and points out the lack of systematized data collection methods at present as well as the feasibility of producing reliable estimates to guide effective intervention strategies. Third, building sustainable collaborative relationships between local social work practitioners, INGOs and academic researchers is crucial in supporting capacity building to effectively plan, monitor and report on social development projects. As social work in many countries becomes increasingly alert to its international dimension (Lyons, 2006; 
Kindler, 2007), our pilot study demonstrates how INGOs, academic researchers, and local social work practitioners can work collaboratively in a way that promotes ethical and evidence informed practice strategies in local contexts. 


\section{Reference}

Alderson P and Morrow V (2011) The Ethics of Research with Children and Young People: A Practical Handbook. London: Sage Publication.

Baker AJ and Charvat BJ (2008) Research Methods In Child Welfare. New York: Columbia University Press.

Bartunek JM and Rynes SL (2014) Academics and Practitioners Are Alike and Unlike. Journal of Management 40(5): 1181-1201.

Belfrage S (2016) Exploitative, irresistible, and coercive offers: why research participants should be paid well or not at all. Journal of Global Ethics 12(1): 69-86.

Belkindas MV and Swanson EV (2014) International support for data openness and transparency. Statistical Journal of the IAOS 30(2): 109-112.

Bhatta CD (2013) External Influence and Challenges of State-building in Nepal. Asian Journal of Political Science 21(2): 169-188.

Deane T (2010) Cross-Border Trafficking in Nepal and India-Violating Women's Rights. Human Rights Review 11(4): 491-513.

ECPAT Luxembourg \& Child Workers in Nepal Concerned Centre (2015) Preparatory Study for Situational Analysis of Commercial Sexual Exploitation of Children in Nepal.

Embassy of the United States, Kathmandu (2016) 2016: Trafficking in Persons Report. Available at: https://nepal.usembassy.gov/2016-trafficking-inpersons-report.html (accessed 10 August 2017).

Frederick J, Basnyat M and Aguettant J (2010) Trafficking and Exploitation in The Entertainment and Sex Industries in Nepal: A Handbook For Decision-makers. Kathmandu, Nepal: Terre des hommes Foundation. 
Grant R and Sugarman J (2004) Ethics in human subjects research: Do incentives matter? Journal of Medicine and Philosophy 29(6): 717-738.

Holmstrom D (1999) Saving Nepal's girls. Available at: http://www.csmonitor.com/1999/0414/p11s1.html (accessed 2 August 2017).

Harwin J and Barron T (2007) The role of service delivery nongovernmental organisations in policy reform. Child Abuse Review 16(6): 367-382.

ILO / Kutnick B, Belser P and Danailva-Trainor G (2007) Methodologies for Global and National Estimation of Human Trafficking Victims: Current and Future Approaches. Geneva: International Labour Office.

Johnston LG and Sabin K (2010) Sampling hard-to-reach populations with respondent driven sampling. Methodological Innovations Online 5(2): 38-48.

Johnston LG, Thurman TR, Mock N, Nano L and Carcani V (2010). Respondent-driven sampling: A new method for studying street children with finds from Albania. Vulnerable Children \& Youth Studies 5(1): 1-11.

Kindler H (2007) Developing evidence-based child protection practice: A view from Germany. Research on Social Work Practice 18(4): 319-324.

Kolucki B and Lemish D (2011) Communicating with children: Principles and practices that nurture, inspire, excite, educate and heal. Available at: https://www.unicef.org/cwc/files/CwC_Web(2).pdf (accessed 4 September 2017)

Lyons K (2006) Globalization and social work: International and local implications. British Journal of Social Work 36(3): 365-380.

MacIntyre G and Paul S (2013). Teaching Research in Social Work: Capacity and Challenge. British Journal of Social Work 43(4): 685-702. 
Mathur S, Malhotra A and Mehta M (2001) Adolescent girls' life aspirations and reproductive health in Nepal. Reproductive Health Matters 9(17): 91-100.

McCreesh N, Frost SD, Seeley J, Katongole J, Tarsh MN, Ndunguse R, Jichi F, Lunel NL, Maher D, Johnston LG, Sonnenberg P, Copas AJ, Hayes RJ and White RG (2012) Evaluation of respondent-driven sampling. Epidemiology 23(1): 138-147.

O'Kane C (2002) Marginalized children as social actors for social justice in South Asia. British Journal of Social Work 32(6): 697-710.

Pawar M (2015) Action research on social work knowledge creation and dissemination from the Global South. British Journal of Social Work 45(4): 1357-1364.

Regehr C, Stern S and Shlonsky A (2007) Operationalizing evidence-based practice: The development of an institute for evidence-based social work. Research on Social Work Practice 17(3): 408-416.

Rigby P and Whyte B (2015) Children's narrative within a multi-centred, dynamic ecological framework of assessment and planning for child trafficking. British Journal of Social Work 45(1): 34-51.

Sacouman N (2012) Paths of local development: culture, context, power, and the role of nongovernmental organizations. Voluntas: International Journal of Voluntary \& Nonprofit Organizations 23(4): 899-919.

Salganik MJ (2006) Variance estimation, design effects, and sample size calculations for respondent-driven sampling. Journal of Urban Health 83: 98- 111.

Spies GM, Delport CSL and le Roux MP (2015) Developing safety and risk assessment tools and training materials: A research-practice dialogue. Research on Social Work Practice 25(6): 670-680. 
Suárez D and Marshall J (2014) Capacity in the NGO sector: Results from a national survey in Cambodia. Voluntas: International Journal of Voluntary \& Nonprofit Organizations 25(1): 176-200.

Terre des Hommes (n.d.) Tdh Nepal position paper on commercial sexual exploitation of children. Available at: http://endcsecnepal.com/tdhinitiative/ (accessed 1 September 2017)

Ulvila M and Hossain F (2002) Development NGOs and political participation of the poor in Bangladesh and Nepal. Voluntas: International Journal of Voluntary \& Nonprofit Organizations 13(2): 149-163.

UNICEF (2011) Developing Capacities To Realize The Rights of Children and Women: Selected Innovations and Lessons Learned From UNICEF-assisted Programmes. New York: UNICEF.

World Health Organization (2016) INSPIRE: Seven Strategies For Ending Violence Against Children. Geneva: World Health Organization. 
Table 1. Descriptive characteristics of male and female children involved in CSEC

\begin{tabular}{|c|c|c|}
\hline & $\begin{array}{l}\text { Males } \\
\text { N (\%) } \\
\end{array}$ & $\begin{array}{c}\text { Females } \\
\text { N }(\%)\end{array}$ \\
\hline \multicolumn{3}{|l|}{ Age } \\
\hline 18 & $29(30.9)$ & $18(15.0)$ \\
\hline 17 & $47(50.0)$ & $57(47.5)$ \\
\hline 16 & $12(12.8)$ & $29(24.2)$ \\
\hline 15 & $3(3.2)$ & $10(8.3)$ \\
\hline 14 & - & $3(2.5)$ \\
\hline 13 & - & $1(0.8)$ \\
\hline 12 & $1(1.1)$ & $2(1.7)$ \\
\hline \multicolumn{3}{|l|}{ Occupation } \\
\hline Unemployed & $14(14.9)$ & - \\
\hline Full-time & $15(16.0)$ & $100(100.0)$ \\
\hline Part-time & $13(13.8)$ & - \\
\hline Casual/occasional & $12(12.8)$ & - \\
\hline Other & $40(42.6)$ & - \\
\hline \multicolumn{3}{|l|}{ Living arrangement } \\
\hline Living alone & $14(14.9)$ & $17(14.2)$ \\
\hline Living with family & $25(26.6)$ & $20(16.7)$ \\
\hline Living with relative & $9(9.6)$ & $14(11.7)$ \\
\hline Living with friends & 45 (47.9) & $56(46.7)$ \\
\hline $\begin{array}{l}\text { Living with husband/ partner/ } \\
\text { boyfriend }\end{array}$ & $1(1.1)$ & $4(3.3)$ \\
\hline No stable living arrangement & - & $1(0.8)$ \\
\hline Other & - & $8(6.7)$ \\
\hline
\end{tabular}


Table 2. Characteristics of male children involved in CSEC ( $\mathrm{N}=94)$

\begin{tabular}{lc}
\hline & Males \\
& N (\%) \\
\hline Gender and exclusivity of sex partners & $37(39.4)$ \\
Any male & $16(17.0)$ \\
Any female & $37(39.4)$ \\
Only male & $2(2.1)$ \\
Only female & \\
Knowledge of men who have sex with other boys & \\
(MSM) & $13(13.8)$ \\
A lot & $53(56.4)$ \\
A fair bit & $16(17.0)$ \\
Only a bit & $12(12.8)$ \\
No knowledge & \\
\hline
\end{tabular}


Table 3. Characteristics of female children involved in CSEC $(\mathrm{N}=120)$

\begin{tabular}{lc}
\hline & Females \\
& $\mathbf{N}(\mathbf{\%})$ \\
\hline Work place & \\
Dance bars & $52(43.3)$ \\
Cabin restaurant & $7(5.8)$ \\
Dohori restaurant & $24(20.0)$ \\
Dohori sanjh & $2(1.7)$ \\
Tea shop & $2(1.7)$ \\
Bahtti Pasals/ local liquor shop & $12(10.0)$ \\
Massage parlour & $5(4.2)$ \\
Guest house & $13(10.8)$ \\
Other & $3(2.5)$ \\
Types of sexual work activities reported & \\
Talk/joking & \\
Flirting & $29(24.2)$ \\
Kissing/cuddling & $45(37.5)$ \\
Deep kissing & $51(42.5)$ \\
Sensual massage & $52(43.3)$ \\
Intimate touching & $13(10.8)$ \\
Masturbation & $109(90.8)$ \\
Give oral sex & $36(30.0)$ \\
Bullying & $3(2.5)$ \\
Sexual harassment & $6(5.0)$ \\
Using drugs & $22(18.3)$ \\
Erotic dancing & $25(20.8)$ \\
Get oral sex & $16(13.3)$ \\
Vaginal intercourse & $5(4.2)$ \\
Any CSEC & $28(23.3)$ \\
\hline Note: ${ }^{1}$ Participants can & $116(96.7)$ \\
\hline
\end{tabular}

Note: ${ }^{1}$ Participants can choose multiple answers. The answers are not mutually exclusive; ${ }^{2}$ "Any CSEC" was created based on answer categories: intimate touching, masturbation, give oral sex, get oral sex and vaginal intercourse. 